\title{
Introduction of a Hydroxy Group at the para Position and N-Iodophenylation of $N$-Arylamides Using Phenyliodine(III) Bis(trifluoroacetate)
}

Naoki Itoh, Takeshi Sakamoto, Etsuko Miyazawa, and Yasuo Kikugawa*

Faculty of Pharmaceutical Sciences, Josai University, 1-1 Keyakidai, Sakado, Saitama

$$
\text { 350-0295, Japan }
$$

\section{Supporting Information}


167-168 ${ }^{\circ} \mathrm{C}$ (lit. ${ }^{3)} 165-166^{\circ} \mathrm{C}$ ); $1 \mathbf{k}: \mathrm{mp} 143-144^{\circ} \mathrm{C}\left(\right.$ lit. $\left.{ }^{4)} 135-136{ }^{\circ} \mathrm{C}\right)$; $\mathbf{1 q}: \mathrm{mp} 182-183^{\circ} \mathrm{C}$ (lit. ${ }^{5)} 181-182{ }^{\circ} \mathrm{C}$ ); 1 r: $\mathrm{mp} 82-83{ }^{\circ} \mathrm{C}\left(\right.$ (lit. ${ }^{6} 84^{\circ} \mathrm{C}$ ); 1 s: $\mathrm{mp} 102-103{ }^{\circ} \mathrm{C}$ (lit. $\left.{ }^{6)} 103-104{ }^{\circ} \mathrm{C}\right) ; \mathbf{1 t}$ : mp 90-92 ${ }^{\circ} \mathrm{C}$ (lit. ${ }^{7} 89.5-90.5^{\circ} \mathrm{C}$ ); 1u: mp $65-66^{\circ} \mathrm{C}$ (lit. ${ }^{8)} 67-68^{\circ} \mathrm{C}$ ); 1v: mp 126-126.5 ${ }^{\circ} \mathrm{C}$ (lit. $\left.{ }^{9}\right) 123-124.5^{\circ} \mathrm{C}$ ); $2 \mathbf{a}: \mathrm{mp} 164-167^{\circ} \mathrm{C}$ (lit. ${ }^{10)} 166-167^{\circ} \mathrm{C}$ ); $\mathbf{2 b}$ : mp $213-220{ }^{\circ} \mathrm{C}$ (lit. ${ }^{11)}$ 214-216 ${ }^{\circ} \mathrm{C}$ ); 2 c: $\mathrm{mp} 176-177^{\circ} \mathrm{C}$ (lit. ${ }^{12)} 170-170.5^{\circ} \mathrm{C}$ ); 2f: mp $185-187^{\circ} \mathrm{C}$ (lit. ${ }^{13)} 177-178$ ${ }^{\circ} \mathrm{C}$ ); 2 g: mp $126-127^{\circ} \mathrm{C}$ (lit. ${ }^{14)} 121^{\circ} \mathrm{C}$ ); $2 \mathbf{h}: \mathrm{mp} 172-175^{\circ} \mathrm{C}$ (lit. ${ }^{15)} 169-171{ }^{\circ} \mathrm{C}$ ); $\mathbf{2 m}: \mathbf{m p}$ 176-179 ${ }^{\circ} \mathrm{C}$ (lit. ${ }^{16)} 178^{\circ} \mathrm{C}$ ); 3a: mp $93-95^{\circ} \mathrm{C}$ (lit..$^{17)} 90^{\circ} \mathrm{C}$ ); $\mathbf{4 b}: \mathrm{mp} 175-177{ }^{\circ} \mathrm{C}$ (lit. ${ }^{18)}$ 165-166.5 ${ }^{\circ} \mathrm{C}$ ); 5a: mp $260-263{ }^{\circ} \mathrm{C}$ (lit. ${ }^{19)} 266-268^{\circ} \mathrm{C}$ ); 5b: mp $237-238^{\circ} \mathrm{C}$ (lit. ${ }^{20)} 237-238$ $\left.{ }^{\circ} \mathrm{C}\right)$; 5c: mp $210-213{ }^{\circ} \mathrm{C}\left(\right.$ lit. ${ }^{21)} 209-210{ }^{\circ} \mathrm{C}$ ); 5d: mp $249-252^{\circ} \mathrm{C}$ (lit. ${ }^{22)} 249-250{ }^{\circ} \mathrm{C}$ ).

\section{References}

(1) Simons, J. H.; Fleming, G. H.; Whitmore, F. C.; Bissinger, W. E. J. Am. Chem. Soc. 1938, 60, 2267.

(2) Schoenberg, A.; Heck, R. F. J. Org. Chem. 1974, 39, 3327.

(3) Clarl, C. R.; Mcmillian, C. L. J. Pharm. Sci. 1990, 79, 220.

(4) Auricchio, S.; Bini, A.; Pastormerlo, E.; Ricca A.; Truscello, A. M. Tetrahedron 1994, 50,7589 .

(5) Nagarajan, V.; Paulus, H.; Weiden, N.; Weiss, A. J. Chem. Soc., Faraday Trans. 2 1986, 
$82,1499$.

(6) Chaplin, H. O.; Hunter, L. J. Chem. Soc. 1938, 375.

(7) Reed, Jr., R. J. Am. Chem. Soc. 1956, 78, 801.

(8) Rutherford, K. G.; Ing, S. Y.-S.; Thibert, R. J. Can. J. Chem. 1965, 43, 541.

(9) Bourne, E. J.; Henry, S. H.; Tatlow, C. E. M.; Tatlow, J. C. J. Chem. Soc. 1952, 4014.

(10) Hewitt, J. T.; Ratcliffe, W. H. J. Chem. Soc. 1912, 101, 1765.

(11) LeRosen, A. L.; Smith, E. D. J. Am. Chem. Soc. 1948, 70, 2705.

(12) Dai, W.-M.; Cheung, Y. K.; Tang, K. W.; Choi, P. Y.; Chung, S. L. Tetrahedron 1995, $51,12263$.

(13) Kesten, S. J.; Johnson, J.; Werbel, L. M. J. Med. Chem. 1987, $30,906$.

(14) Hurst, W. G.; Thorpe, J. F. J. Chem. Soc. 1915, 107, 934.

(15) Heidelberger M.; Jacobs W. A. J. Am. Chem. Soc. 1919, 41, 1450.

(16) Kehrmann, F.; Kissine, D. Ber. 1914, 47, 3096.

(17) Fehlhaber, H.-W.; Whlzel, P. Org. Mass. Spectrom. 1970, 4, 545.

(18) Blout, E. R.; Silverman, D. C. J. Am. Chem. Soc. 1944, 66, 1442.

(19) Sakamoto, T.; Hosoda, I.; Kikugawa, Y. J. Heterocycl. Chem. 1988, 25, 1279.

(20) Mayer, F.; Zütphen, L.; Philipps, H. Ber. 1927, 60, 858.

(21) Zinner, H.; Wigert, H. Chem. Ber. 1960, 93, 1331.

(22) Loudon, J. D.; Ogg, J. J. Chem. Soc. 1955, 739. 